\title{
SOLVABILITY OF CONVOLUTION EQUATIONS IN $\mathcal{K}_{1}^{\prime}$
}

\author{
S. SZNAJDER AND Z. ZIELEZNY
}

\begin{abstract}
Let $S$ be a convolution operator in the space $\mathscr{K}_{1}{ }^{\prime}$ of distributions of exponential growth. A condition on $S$ introduced by $O$. von Grudzinski is proved to be equivalent to $S * \mathscr{K}_{1}^{\prime}=\mathscr{K}_{1}^{\prime}$.
\end{abstract}

This paper is motivated by a recent result of $O$. von Grudzinski [2], who characterized convolution operators in $\mathscr{D}^{\prime}$ having fundamental solutions of exponential growth in $R^{n}$. Convolution operators in the space $\mathscr{D}^{\prime}$ of all distributions in $R^{n}$ are distributions with compact support, i.e. in $\mathcal{E}^{\prime}$. We state the main part of Grudzinski's theorem (see [2, Theorem 1.1]) in a form suitable for our purpose, using the space $\mathcal{K}_{1}^{\prime}$ of distributions of exponential growth introduced by M. Hasumi [3].

Let $S$ be a distribution in $\mathcal{E}^{\prime}$ and $\hat{S}$ its Fourier transform. The following conditions are equivalent:

(a) There exist positive constants $N, r, C$ such that

$$
\sup _{s \in C^{n},|s| \leqslant r}|\hat{S}(\xi+s)| \geqslant \frac{C}{(1+|\xi|)^{N}}, \quad \xi \in R^{n}
$$

(b) $S$ has a fundamental solution in $\mathscr{K}_{1}{ }^{\prime}$.

We recall that a distribution $E \in \mathscr{D}^{\prime}$ is a fundamental solution for $S \in \mathcal{E}^{\prime}$ if $S * E=\delta$ where * denotes the convolution and $\delta$ the Dirac measure at the origin.

We now ask the question of solvability of convolution equations in $\mathcal{K}_{1}^{\prime}$. Let $\mathcal{O}_{C}^{\prime}\left(\mathcal{K}_{1}^{\prime}: \mathscr{K}_{1}^{\prime}\right)$ be the space of convolution operators in $\mathscr{K}_{1}^{\prime}$ (see [3] or [5]). Under what conditions on $S \in \mathcal{O}_{C}^{\prime}\left(\mathcal{K}_{1}^{\prime}: \mathscr{K}_{1}^{\prime}\right)$ is $S * \mathcal{K}_{1}^{\prime}=\mathscr{K}_{1}^{\prime}$ ? The last equality means that the mapping $u \rightarrow S * u$ maps $\mathscr{K}_{1}^{\prime}$ onto $\mathcal{K}_{1}^{\prime}$.

THEOREM. If $S$ is a distribution in $\mathcal{O}_{C}^{\prime}\left(\mathcal{K}_{1}^{\prime}: \mathcal{K}_{1}^{\prime}\right)$ then each of the conditions (a) and $(\mathrm{b})$ is equivalent to $(\mathrm{c}) S * \mathcal{K}_{1}^{\prime}=\mathscr{K}_{1}^{\prime}$.

Before presenting the proof we recall the basic facts about the spaces $\mathscr{K}_{1}^{\prime}$ and $\vartheta_{C}^{\prime}\left(\mathcal{K}_{1}^{\prime}: \mathscr{K}_{1}^{\prime}\right)$; the proofs can be found in [3] or [5].

Let $\mathscr{K}_{1}$ be the space of all $C^{\infty}$-functions $\varphi$ in $R^{n}$ such that

$$
v_{k}(\varphi)=\sup _{x \in R^{n},|\alpha| \leqslant k} \gamma_{k}(x)\left|D^{\alpha} \varphi(x)\right|<\infty, \quad k=0,1, \ldots,
$$

Received by the editors July 7, 1975.

AMS (MOS) subject classifications (1970). Primary 45E10; Secondary 46F99.

- American Mathematical Society 1976 
where $\quad D^{\alpha}=D_{1}^{\alpha_{1}} \cdots D_{n}^{\alpha_{n}}, D_{j}=i^{-1} \partial / \partial x_{j}$ and $\gamma_{k}(x)=\prod_{j=1}^{n}\left(e^{k x_{j}}+e^{-k x_{j}}\right)$. The topology in $\mathscr{K}_{1}$ is defined by the seminorms $v_{k}$. Then $\mathscr{K}_{1}$ is a Fréchet space.

The dual $\mathcal{K}_{1}^{\prime}$ of $\mathscr{K}_{1}$ is the space of distributions of exponential growth. A distribution $u \in \mathscr{D}^{\prime}$ is in $\mathcal{K}_{1}^{\prime}$ if and only if there exists a multi-index $\beta$, an integer $k \geqslant 0$ and a bounded, continuous function $f$ in $R^{n}$ such that $u=D^{\beta}\left(\gamma_{k} f\right)$. $\mathscr{K}_{1}^{\prime}$ is endowed with the topology of uniform convergence on all bounded sets in $\mathscr{K}_{1}$.

If $u \in \mathscr{K}_{1}^{\prime}$ and $\varphi \in \mathscr{K}_{1}$, then the convolution $u * \varphi$ is a $C^{\infty}$-function defined by

$$
u * \varphi(x)=\left\langle u_{y}, \varphi(x-y)\right\rangle
$$

where $\langle u, \varphi\rangle=u(\varphi)$.

More generally, the space $\mathcal{O}_{C}^{\prime}\left(\mathcal{K}_{1}^{\prime}: \mathscr{K}_{1}^{\prime}\right)$ of convolution operators in $\mathcal{K}_{1}^{\prime}$ consists of distributions $S \in \mathscr{K}_{1}^{\prime}$ satisfying one of the equivalent conditions:

(i) The products $\gamma_{k} S, k=0,1, \ldots$, are tempered distributions.

(ii) Given any $k=0,1, \ldots, S$ can be represented in the form $S$ $=\sum_{|\alpha| \leqslant m} D^{\alpha} f_{\alpha}$, where $f_{\alpha},|\alpha| \leqslant m$, are continuous functions in $R^{n}$ such that

$$
f_{\alpha}(x)=O\left(1 / \gamma_{k}(x)\right) \text { as }|x| \rightarrow \infty .
$$

(iii) For every $\varphi \in \mathscr{K}_{1}, S * \varphi$ is in $\mathscr{K}_{1}$. Moreover, the mapping $\varphi \rightarrow S$ $* \varphi$ of $\mathscr{K}_{1}$ into $\mathscr{K}_{1}$ is continuous.

If $S \in \mathcal{O}_{C}^{\prime}\left(\mathscr{K}_{1}^{\prime}: \mathscr{K}_{1}^{\prime}\right)$ and $\check{S}$ is obtained from $S$ by symmetry with respect to the origin, i.e. $\langle\check{S}, \varphi\rangle=\left\langle S_{x}, \varphi(-x)\right\rangle, \varphi \in \mathcal{K}_{1}$, then $\check{S}$ is also in $\mathcal{O}_{C}^{\prime}\left(\mathcal{K}_{1}^{\prime}: \mathcal{K}_{1}^{\prime}\right)$. The convolution of $S$ with $u \in \Re_{1}^{\prime}$ is then defined by

$$
\langle S * u, \varphi\rangle=\langle u, \check{S} * \varphi\rangle, \quad \varphi \in \mathcal{K}_{1} .
$$

For $\varphi \in \mathcal{K}_{1}$, the Fourier transform

$$
\hat{\varphi}(\xi)=\int_{R^{n}} e^{-i\langle\xi, x\rangle} \varphi(x) d x
$$

can be continued in $C^{n}$ as an entire function such that

$$
w_{k}(\hat{\varphi})=\sup _{\zeta \in C^{n},|\operatorname{Im} \zeta| \leqslant k}(1+|\zeta|)^{k}|\hat{\varphi}(\zeta)|<\infty, \quad k=0,1, \ldots
$$

If $K_{1}$ is the space of all entire functions with property (2) and the topology in $K_{1}$ is defined by the seminorms $w_{k}$, then the Fourier transform is an isomorphism of $\mathscr{K}_{1}$ onto $K_{1}$.

The dual $\mathcal{K}_{1}^{\prime}$ of $K_{1}$ is the space of Fourier transforms of distributions in $\mathcal{K}_{1}^{\prime}$. For $u \in \mathscr{K}_{1}^{\prime}$, the Fourier transform $\hat{u}$ is defined by the Parseval formula

$$
\langle\hat{u}, \hat{\varphi}\rangle=(2 \pi)^{n}\left\langle u_{x}, \varphi(-x)\right\rangle .
$$

The Fourier transform $\hat{S}$ of a distribution $S \in \mathcal{O}_{C}^{\prime}\left(\mathcal{K}_{1}^{\prime}: \mathcal{K}_{1}^{\prime}\right)$ is a function which can be continued in $C^{n}$ as an entire function with the following property: for every $k=0,1, \ldots$, there exists $l=0,1, \ldots$, such that

$$
\sup _{\zeta \in C^{n}, \operatorname{Im} \zeta \mid \leqslant k}|\hat{S}(\zeta)|(1+|\zeta|)^{-l}<\infty .
$$


Also, if $S \in \mathcal{O}_{C}^{\prime}\left(\mathscr{K}_{1}^{\prime}: \mathscr{K}_{1}^{\prime}\right)$ and $u \in \mathcal{K}_{1}^{\prime}$, we have the formula

$$
\widehat{S * u}=\hat{S} \hat{u},
$$

where the product on the right-hand side is defined by $\langle\hat{S} \hat{u}, \chi\rangle=\langle\hat{u}, \hat{S} \chi\rangle, \chi$ $\in K_{1}$.

In the proof of our theorem we shall make use of the following lemma of $L$. Hörmander (see [4, Lemma 3.2]):

If $F, G$ and $F / G$ are entire functions and $\rho$ is an arbitrary positive number, then

$$
|F(\zeta) / G(\zeta)| \leqslant \sup _{|\zeta-s|<4 \rho}|F(s)| \sup _{|\zeta-s|<4 \rho}|G(s)| /\left(\sup _{|\zeta-s|<\rho}|G(s)|\right)^{2},
$$

where $\zeta, s \in C^{n}$.

Proof OF THE THEOREM. It is obvious that $(\mathrm{c}) \Rightarrow(\mathrm{b})$. The implication $(\mathrm{b}) \Rightarrow$ (a) was proved in [2] for $S \in \mathcal{E}^{\prime}$. In the more general case where $S$ $\in \mathcal{O}_{C}^{\prime}\left(\mathcal{K}_{1}^{\prime}: \mathcal{K}_{1}^{\prime}\right)$ the proof of this implication needs only minor modifications and therefore we leave it out. Thus it remains to show that (a) $\Rightarrow$ (c).

Let $S$ be a distribution in $\mathcal{O}_{C}^{\prime}\left(\mathcal{K}_{1}^{\prime}: \mathcal{K}_{1}^{\prime}\right)$ satisfying condition (a) and let $T=\check{S}$; in that case $T$ also satisfies condition (a). We consider the mapping $S *: u \rightarrow S * u$ of $\mathcal{K}_{1}^{\prime}$ into $K_{1}^{\prime}$. By (1), $S *$ is the transpose of the mapping $T *: \varphi \rightarrow T * \varphi$ of $\mathcal{K}_{1}$ into $\mathcal{K}_{1}$. In order to prove (c) it suffices to show that $T *$ is an isomorphism of $\mathscr{K}_{1}$ onto $T * \mathscr{K}_{1}$ (see [1, Corollary, p. 92]).

By what we have said before, the mapping $T *$ is continuous. Also, using Fourier transforms it is easy to see that $T *$ is injective. We now prove that the inverse of $T *$, i.e. the mapping $T * \varphi \rightarrow \varphi$, is continuous. Since the Fourier transformation is an isomorphism, it suffices to prove the equivalent statement that the mapping $\hat{T} \hat{\varphi} \rightarrow \hat{\varphi}$ is continuous.

Suppose that $\hat{T} \hat{\varphi}=\hat{\psi}$ where $\hat{\varphi}, \hat{\psi} \in K_{1}$ and $\hat{T}$ is an entire function satisfying condition (a). We pick an integer $k \geqslant 0$ arbitrarily and assume that $\zeta=\xi+i \eta$ is in the horizontal strip $|\eta| \leqslant k$. Applying to the functions $\hat{\psi}, \hat{T}$ (and $\hat{\psi} / \hat{T}=\hat{\varphi}$ ) Hörmander's Lemma with $\rho=k+r$, we obtain

$$
|\hat{\varphi}(\zeta)| \leqslant \sup _{|\zeta-s|<4(k+r)}|\hat{\psi}(s)| \sup _{|\zeta-s|<\mathbf{4}(k+r)}|\hat{T}(s)| /\left(\sup _{|\zeta-s|<k+r}|T(s)|\right)^{2} .
$$

But

$$
\begin{aligned}
\sup _{|\zeta-s|<k+r}|\hat{T}(s)| & =\sup _{|s|<k+r}|\hat{T}(\zeta+s)| \geqslant \sup _{|s|<r}|\hat{T}(\xi+s)| \\
& \geqslant C /(1+|\xi|)^{N} \geqslant C /(1+|\zeta|)^{N},
\end{aligned}
$$

where we made use of condition (a).

On the other hand, since $|\eta| \leqslant k$, there exist constants $N^{\prime}, C^{\prime}>0$ such that

$$
\sup _{|\zeta-s|<4(k+r)}|\hat{T}(s)|=\sup _{|s|<4(k+r)}|\hat{T}(\zeta+s)| \leqslant C^{\prime}(1+|\zeta|)^{N^{\prime}},
$$

in view of (3).

Now combining (4) with (5) and (6) we obtain 


$$
|\hat{\varphi}(\zeta)| \leqslant C^{\prime \prime}(1+|\zeta|)^{2 N+N^{\prime}} \sup _{|\zeta-s|<4(k+r)}|\hat{\psi}(\zeta)|,
$$

where $C^{\prime \prime}$ is a constant. Hence it follows that

$$
w_{k}(\hat{\varphi}) \leqslant C^{*} w_{l}(\hat{\psi})
$$

where $C^{*}$ is another constant (depending only on $T$ and $k$ ) and $l$ is an integer $\geqslant \max \left\{5 k+4 r, k+2 N+N^{\prime}\right\}$. This proves the continuity of the mapping $\hat{T} \hat{\varphi}=\hat{\psi} \rightarrow \hat{\varphi}$ and consequently the implication $(\mathrm{a}) \Rightarrow(\mathrm{c})$.

\section{REFERENCES}

1. J. Dieudonné and L. Schwartz, La dualité dans les espaces (F) et ([9G), Ann. Inst. Fourier (Grenoble) 1 (1949), 61-101 (1950). MR 12, 417.

2. $\mathrm{O}$. von Grudzinski, Uber Fundamentallösungen von Convolutoren und von DifferentialDifferenzen-Operatoren mit knostanten Koeffizienten, Dissertation, Kiel, 1974.

3. M. Hasumi, Note on the n-dimensional tempered ultra-distributions, Tôhoku Math. J. (2) 13 (1961), 94-104. MR 24 \# A1607.

4. L. Hörmander, On the range of convolution operators, Ann. of Math. (2) 76 (1962), 148-170. MR 25 \#5379.

5. Z. Zieleźny, On the space of convolution operators in $\mathcal{K}_{1}{ }^{\prime}$, Studia Math. 31 (1968), 111-124. MR 40 \# 1772.

Department of Mathematics, State University of New York at Buffalo, Amherst, New YoRK 14226 (Current address of Z. Zielezny)

Current address (S. Sznajder): Department of Mathematics, University of Copenhagen, 2100 Copenhagan, Denmark 